\title{
Pensar el ingreso y el primer año. Un ejercicio de reflexión sobre la experiencia docente en el curso introductorio a las carreras de Sociología - FaHCE/UNLP
}

\author{
Thinking about entrance and first year of university. A reflection exercise on the \\ teaching experience in the introductory course to Sociology careers
}

\author{
Micaela Barrena \\ micabarrena@gmail.com \\ Universidad Nacional de La Plata, Argentina \\ Emilia Di Piero \\ medipiero@gmail.com \\ Universidad Nacional de La Plata, Argentina \\ Pamela Ferroni \\ pamelaferroni82@gmail.com \\ Universidad Nacional de La Plata, Argentina \\ Santiago Garriga Olmo \\ santiago.garriga@hotmail.com \\ Universidad Nacional de La Plata, Argentina \\ Brunela Germán \\ brunelagerman@gmail.com \\ Universidad Nacional de La Plata, Argentina
}

Miguel Kiperszmid

migue.kiper@gmail.com

Universidad Nacional de La Plata, Argentina

Lucía Reartes

lucia.reartes.eliosoff@gmail.com

Universidad Nacional de La Plata, Argentina

\author{
Miguel Reartes \\ miguel.reartes@gmail.com \\ Universidad Nacional de La Plata, Argentina
}

Recepción: 15 Agosto 2020

Aprobación: 01 Octubre 2020

Publicación: 01 Diciembre 2020
Resumen: En este trabajo reflexionamos sobre nuestra experiencia como docentes del Curso Introductorio a las carreras de Profesorado y Licenciatura en Sociología de la Facultad de Humanidades y Ciencias de la Educación perteneciente a la Universidad Nacional de La Plata. En primer lugar, desarrollamos aportes teóricos sobre la importancia de abordar el ingreso a los estudios superiores como tema de agenda, luego describimos las características estructurantes del curso, y, por último, los abordajes pedagógicos y las actividadesimplementadas por el cuerpo docente. Finalmente, presentamos preguntas y desafíos pendientes que emergen de las reflexiones colectivas y balances sobre la experiencia docente.

Palabras clave: Ingreso, Sociología, Experiencia docente, Democratización.

Abstract: This work retrieves our experiences and reflections as teaching staff of the Introductory Course of the Undergraduate and Teaching Degrees in Sociology at the Facultad de Humanidades y Ciencias de la Educación, Universidad Nacional de La Plata. First of all, the theoretical contributions on the importance of studying the entrance into higher studies are reconstructed; then, the structuring characteristics of the course and, lastly, the pedagogical approaches and activities implemented by the teaching staff during the course. Finally, the questions and challenges that emerge from the collective reflections and balances on the teaching experience are presented.

Keywords: University entrance, Sociology, Teaching experience, Democratization.

Cita sugerida: Barrena, M., Di Piero, E., Ferroni, P., Garriga Olmo, S., Germán, B., Kiperszmid, M.,... Reartes, M. (2020). Pensar el ingreso y el primer año. Un ejercicio de reflexión sobre la experiencia docente en el curso introductorio a las carreras de Sociología - FaHCE/UNLP. Cuestiones de Sociología, 23, e108. https://doi.org/10.24215/23468904e108 


\section{INTRODUCCIÓN: SOBRE EL INGRESO Y EL PRIMER AÑO EN LAS UNIVERSIDADES ARGENTINAS}

En este trabajo reflexionamos en torno al ingreso a la universidad recuperando nuestra experiencia como docentes del Curso Introductorio a las carreras de Profesorado y Licenciatura en Sociología de la Facultad de Humanidades y Ciencias de la Educación perteneciente a la Universidad Nacional de La Plata.En primer lugar, desarrollamos aportes teóricos que contextualizan la importancia de abordar la cuestión del ingreso a los estudios superiores; luego describimos las características estructurantes del curso, y, por último, analizamos y reflexionamos sobre las actividades y prácticas implementadas por el cuerpo docente durante el transcurso del mismo.

La cuestión del ingreso y el primer año en el nivel superior fue ganando relevancia, tanto en el campo de estudios sobre educación como entre las políticas institucionales, en la medida en que el nivel se fue masificando. Chiroleu (1998) indica que desde la década del 50 ha tenido lugar un crecimiento continuo y sin precedentes de la matrícula en educación superior mundial. Esta tendencia masificó este nivel del sistema educativo y dio lugar "al tránsito de una universidad de élites a un modelo de acceso de masas, tránsito que por otra parte tuvo impacto en materia de democratización, ya que facilitó la incorporación de sectores previa y tradicionalmente excluidos" (Chiroleu, 1998, p. 4).

Es en ese marco que se reposicionan las preguntas en torno al desgranamiento en el primer año, tanto desde las indagaciones académicas como desde las preocupaciones y políticas a nivel institucional. En ese sentido, más allá del carácter abierto y amplio del nivel universitario en Argentina, entre lxs ingresantes al nivel superior es posible encontrar una diversidad de trayectorias, que muchas veces implica también cierta desigualdad: cuentan con diferentes puntos de partida en relación con sus socializaciones familiares, sus redes de sociabilidad previas, sus saberes, y, sobre todo, con las escuelas secundarias por las que transitaron.

Los estudios sobre la problemática del ingreso y la permanencia en los estudios superiores universitarios muestran que, más allá de la masificación del acceso, se produce una selección implícita diferida, que da lugar a una "inclusión excluyente" (Ezcurra, 2011). En relación con ello emergen las problemáticas de la retención y la permanencia ligadas, entre otras cuestiones, al acceso de lxs estudiantes a una cultura peculiar, la académica, que tiene entre sus rasgos centrales su carácter escrito. Los desiguales puntos de partida de lxs ingresantes en lo que refiere a la relación con dicha cultura inciden en la capacidad de permanecer ligada a una resocialización más o menos cercana a las socializaciones familiares y escolares previas. ${ }^{1}$

Esta selección no emerge solo en relación con el nivel socioeconómico (problema que se resolvería parcialmente mediante el otorgamiento de becas), sino también en relación con condicionantes académicos y culturales previos. En ese sentido, en el último tiempo se propone una serie de dispositivos institucionales que buscan igualar los puntos de partida; entre otros, los cursos de ingreso con carácter introductorio. Para ese propósito también adquiere relevancia la práctica y el rol docente, ya que "la forma en que se produce y distribuye el conocimiento entre los ingresantes adquiere aquí centralidad en términos pedagógicos, pero también políticos" (Pierella, 2018, p. 172). 


\section{Sobre el Curso Introductorio a Sociología - FAHCE/UNLP}

El Curso Introductorio a las carreras de Sociología de la FaHCE/UNLP, implementado desde el año 2012 y con carácter obligatorio desde el año 2016, constituye una instancia más entre las políticas de inclusión a la educación superior. Lejos de toda finalidad selectiva, en cada una de las nueve ediciones del Curso se aspiró a enseñar lo omitido, es decir, a explicitar ciertos saberes que son parte de la vida universitaria y que suelen darse por supuestos.

El equipo docente se compone en la actualidad de una coordinadora general, siete docentes y catorce colaboradorxs estudiantes. Cada año se conforman siete comisiones, integradas por un docente y dos colaboradorxs, para el trabajo intensivo a lo largo de un mes, desde inicios de febrero. Alrededor de doscientos estudiantes realizan el curso anualmente. Las siete comisiones de trabajo funcionan a diario distribuidas en tres bandas horarias durante el transcurso de un mes: de 8 a 10 horas (dos comisiones), de 10 a 12 horas (tres comisiones) y de 18 a 20 horas (dos comisiones). Asimismo, las carreras de Sociología cuentan con estudiantes privadxs de la libertad que se encuentran alojadxs en los penales de La Plata y sus alrededores. Desde el año 2017, los equipos docentes dictan un taller en el cual se trabaja la perspectiva propia de la Sociología, así como también comunican la información central sobre la carrera. Se realiza en el marco de uno a dos encuentros y se asiste aproximadamente a 4 cárceles - de varones y de mujeres- junto al Programa de Acompañamiento a Estudiantes Privados/as de la Libertad de la Facultad.

De esta manera, el curso ocuparía el lugar de una asignatura ad hoc orientada al desarrollo progresivo de ciertas disposiciones, habilidades, hábitos y redes de sociabilidad, que favorecerían la retención y el desarrollo de una "afiliación" (Coulon, 1995) que buscar aportar a la construcción del oficio de estudiante universitarix, así como de una "alfabetización" académica. Por esta última se entiende

el conjunto de nociones y estrategias necesarias para participar en las culturas discursivas de las disciplinas, así como en la producción y análisis de textos requeridos para aprender en la universidad. Apunta, de esta manera, a las prácticas de lenguaje y pensamiento propias del ámbito académico superior (Carlino, 2005, p. 13).

La experiencia ha demostrado que se abre una brecha entre el tipo de estudiante esperado y el real, y que aparecen dificultades bien "por desconocimiento" o bien por trayectorias reales que -tendencialmente concentradas en las comisiones del turno tarde en el caso del curso- se distancian de las trayectorias teóricas (Terigi, 2009). Quienes finalmente logran los aprendizajes necesarios lo hacen solxs, o por ensayo y error. El Curso Introductorio a Sociología aspira a explicitar esos saberes implícitos, a evitar esa "enseñanza omitida en los programas de las asignaturas y también en los planes de estudio" (Ezcurra, 2011, p. 63).

Otra dimensión que genera y reproduce desigualdades refiere a las redes de sociabilidad. Este aspecto resulta aún más relevante si evaluamos que, según el estudio de Rossi Casé, Aguiar y Varela (2006), en el año 2004 el 52\% de lxs ingresantes a Sociología provenían de localidades diferentes al Gran La Plata. Este porcentaje es ratificado por Cotignola, Legarralde y Margueriche (2018), quienes para los años 2014-2018 indican que

la mitad de los alumnos que ingresan son residentes de la ciudad de La Plata. De aquellos que residen en partidos circundantes, las/os ingresantes proceden principalmente de Berisso (2,4\%), Ensenada (2,2\%), Berazategui (1,9\%), Quilmes (1,7\%) y Florencio Varela (1\%).

Ciertamente, se trata de una población que en buena medida no proviene del Gran La Plata y zonas circundantes, por lo cual no cuenta con redes personales preestablecidas. Esto implica que resulte central que la experiencia en el curso habilite lazos y fabrique "soportes" que lxs envuelvan en relaciones sociales que cooperen con sostenerlos. En ese sentido, es sustancial tender a que el número de estudiantes por comisión sea acotado, de modo de habilitar la proximidad de los vínculos en una escala humanizada, que permita a lxs docentes "conectar con lxs estudiantes", y a lxs estudiantes conectarse entre sí.

En una exploración previa se ha mostrado que existen 29 carreras de Sociología en el nivel universitario en Argentina, y se han sistematizado sus políticas de admisión (Di Piero, 2019). De ese modo, en el 
mencionado trabajo se indicó que presentan un mapa variopinto en lo referido a las políticas de admisión, en las que se conjugan objetivos diversos: familiarización (1), nivelación (6), incorporación de contenidos (4), ambientación (3), sociabilidad (2), introducción (1), vocacional (1), diagnóstico (1) y pago (1). Sin embargo, ninguna de las opciones (exceptuando el pago) se configura como eliminatoria, aunque cabe aclarar que en algunos casos el hecho de no haber aprobado el ingreso implica la imposibilidad de cursar completamente el primer año de la carrera (el primer año queda reducido a algunas materias en particular) (Di Piero, 2019). En el caso del ingreso a Sociología de la FaHCE/UNLP, los objetivos apuntan a la familiarización, la ambientación, la sociabilidad, la introducción y la nivelación. En relación con esos objetivos, los ejes estructurantes de las actividades del curso son los cuatro que mencionamos a continuación.

El primero, "lectura, comprensión y producción de textos", se aboca fundamentalmente a la lectura y comprensión de textos académicos sencillos vinculados a la disciplina y a la producción de textos académicos también sencillos (organización de las ideas, argumentación, redacción), exposición oral y debate argumentado. Al mismo se le destina una carga de 17 horas (que representa el 47,2\% del total del curso).

El segundo eje, "ambientación a la vida universitaria", busca abordar y ofrecer a lxs estudiantes el conocimiento sobre: a) aspectos organizativos y administrativos del Departamento de Sociología y de la Facultad; y b) becas y servicios estudiantiles provistos por la Universidad. El mismo se desarrolla en 13 horas (que representan el 36,2\% del total del curso).

El tercer eje, "gobierno universitario y participación político-institucional estudiantil”, aborda y despliega el marco normativo universitario nacional, de la Universidad Nacional de La Plata y de la Facultad y da cuenta de los espacios de participación y organización estudiantil, desde la estructura del cogobierno universitario hasta los espacios de participación al nivel de la Facultad. Se desarrolla en una carga horaria de 3 horas (8,3\% del curso).

El cuarto eje, "plan de estudios de la carrera e inserción ocupacional del/de la sociólogx", busca abordar: a) el campo disciplinar de la sociología en tanto ciencia social; b) los planes de estudio de la Licenciatura y del Profesorado en Sociología, y c) la inserción ocupacional del/de la sociólogx y el sentido de la práctica profesional. Se desarrolla en 3 horas ( $8,3 \%$ del curso).

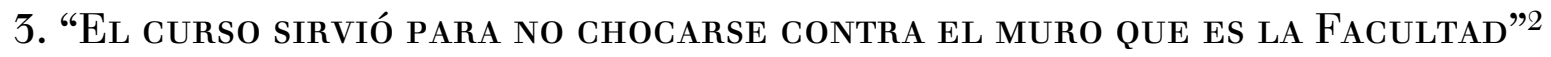

Entendemos que las actividades del curso dan lugar a una práctica docente particular, con especificidades que la diferencian tanto de la docencia secundaria como de la práctica docente habitual en las universidades. Dos condiciones estructurales habilitan la singularidad de esa práctica: por un lado, el encuentro con lxs estudiantes es cotidiano, y, por otro, las comisiones cuentan con un número relativamente pequeño de estudiantes. Ambas pautas dan lugar a la conformación de vínculos más estrechos, cara a cara, personalizados. A su vez, a partir de esa experiencia se ponen en juego dinámicas áulicas algo más desestructuradas, que dan margen a la innovación didáctica por parte de lxs docentes. En ese sentido, la instancia del curso es propicia para la incorporación de herramientas y dinámicas propias de las propuestas de formato taller, o bien, en algunos casos, de la educación no-formal. Se trata de actividades que proponen otra disposición de los espacios, que rompe esquemas áulicos tradicionales en pos de generar una condición activa entre los estudiantes. Estas características, junto con un fuerte impulso del trabajo grupal, forman parte del repertorio que lxs docentes ponemos en juego buscando generar tramas de sociabilidad, socialización y espacios de circulación de saberes que trasciendan la duración del curso.

Un ejemplo de ello son los ejercicios para la división en grupos orientados a fomentar la rotación permanente de lxs ingresantes, los cuales proponen grupos diversos donde circulan saberes propios de las diferentes biografías y trayectorias escolares. Estos espacios grupales intencionalmente rotativos buscan favorecer la circulación de la palabra y evitar que en el aula cristalicen tempranamente grupos hacia su interior homogéneos (dinámica de "cierre social” que muchas veces conlleva que lxs más aventajadxs se junten entre 
sí y los menos aventajadxs lo repliquen, obturando el intercambio), al tiempo que permiten volver visibles algunos saberes implícitos (relacionados con los modos de evaluación en la universidad, las diferencias entre un examen final y un parcial, o entre una clase teórica y una práctica, por brindar algunos ejemplos), compartir expectativas y afrontar miedos en relación con el inicio de la experiencia universitaria y la incorporación a la cultura académica.

Sin embargo, también surgen dificultades a la hora de pensar las herramientas que promuevan una mejor articulación entre las experiencias del nivel secundario y el superior. Una de ellas es la tensión a la que se enfrenta el equipo docente entre trabajar a partir de recursos pedagógicos que resulten familiares en relación con las trayectorias previas de lxs ingresantes, y, al mismo tiempo, evitar una excesiva escolarización, ajena a la cultura académica propia de la vida en la universidad. En concordancia con ello, se espera que el curso no sea una "isla" en relación con las materias y las prácticas del primer año y los años subsiguientes, sino que se busca propiciar una transición gradual. En ese sentido, se propone el trabajo desde un paradigma que combina dinámicas "anticipatorias" de aquellas que caracterizarán la experiencia áulica propia de la universidad (clase teórica, toma de apuntes, simulacro de parcial), con dinámicas de seguimiento próximo de los aprendizajes (trabajos con técnicas de lectura, escritura y reescritura), acompañamiento y promoción de la sociabilidad entre pares.

Considerando los ejes anteriormente mencionados, a continuación desarrollamos las actividades realizadas en el curso y las decisiones pedagógicas en las que se enmarcan.

\title{
3.1. Trabajo sobre la lectura, comprensión y producción de textos
}

\author{
"El concepto de texto definitivo no corresponde \\ sino a la religión o al cansancio" (Borges, 1985, p. 95).
}

Uno de los ejes centrales de trabajo en el curso refiere a la lectura, comprensión y producción de textos. En función de ello abordamos siete textos reunidos en un único cuadernillo. ${ }^{3}$ La selección de los textos emergió de un intercambio colaborativo al interior del equipo en el que se pusieron en discusión criterios que exceden lo curricular, dado que el curso no tiene un propósito contenidista que implique la acreditación de saberes o temas específicos (a diferencia de lo que sucede en la mayor parte de las cátedras).

Por una parte, la selección consideró el propósito de trabajar sobre textos que permitieran el entrenamiento de distintas técnicas de estudio: el resumen, el ensayo, el mapa conceptual, el glosario y la línea de tiempo, entre otras. En ese sentido, cada texto fue seleccionado en función de que posibilitara el trabajo con alguna de esas técnicas específicas. Por citar algunos ejemplos, mientras que el abordaje del texto de George Ritzer habilitó la construcción de un glosario y el trabajo de resumen de las ideas principales, a partir del texto de Anthony Giddens se les solicitó que realizaran un ejercicio ensayístico sobre la "imaginación sociológica". Asimismo, los ejercicios de escritura pensados con base en los textos se fueron desarrollando con modalidades diversas: algunos de ellos se realizaron de manera presencial, otros en forma domiciliaria, algunos de modo individual y otros implicaron la escritura grupal. En el mismo sentido, se les solicitó que enviaran uno de los ejercicios vía correo electrónico a lxs profesores, de modo de ejercitar el uso formal de esta vía de comunicación.

Por otra parte, la selección de textos buscó introducir a lxs estudiantes en los problemas que aborda la Sociología y, particularmente, en la construcción de una mirada sociológica. Asimismo, se optó por trabajar con base en textos que resultaran de estudios sociológicos específicos y contemporáneos, y no en textos de teoría sociológica o pensamiento social general. Esta decisión se debió a que consideramos que de ese modo propiciamos un intercambio más cercano a las expectativas de lxs ingresantes con respecto a los contenidos que esperan encontrar en la carrera, al tiempo que impulsamos los primeros pasos hacia la construcción de una 
mirada sociológica. A su vez, lxs docentes seleccionamos algunos de los textos para trabajar en cada comisión en particular, priorizando el análisis en profundidad de cada uno de ellos.

Cada uno de los textos fue trabajado en el aula a partir de objetivos complementarios: comprensión del texto, distinción/ identificación de la mirada sociológica, entrenamiento en herramientas de lectura y abordaje de textos académicos (síntesis, mapeo, subrayado, glosario, notas al margen, mapa conceptual). A su vez, estos objetivos se fueron desagregando, dependiendo de cada texto, a partir del cumplimiento de las siguientes consignas: analizar los argumentos principales; comprender y ejercitar la desnaturalización propia de la perspectiva sociológica; distinguir lxs interlocutores propuestos por lxs autorxs; analizar la estrategia metodológica de cada autor/a; reconocer qué actores aparecen; identificar las variables analizadas; diferenciar la mirada sociológica de otras miradas; distinguir la mirada normativa de la descriptiva o analítica; comprender las particularidades de la sociología y de su relación con otras disciplinas y con el "sentido común"; analizar la articulación entre teoría y empiria propia de la investigación sociológica; reconocer la especificidad formal del texto (artículo académico, sección de una tesis, capítulo de libro, entrevista, nota periodística, etc.).

En lo que concierne a la producción de textos escritos, como equipo docente nos ocupamos de corregir dificultades, y, a la vez, de promover que lxs estudiantes reescribieran sus producciones y mejoraran las sucesivas versiones respetando una idea nodal del curso: la escritura académica entendida como una práctica a entrenar y no como un don o talento natural. De ese modo, la reescritura en tanto entrenamiento se constituye como una dimensión nodal del trabajo en el curso, dado que subrayamos, como sostiene Becker (2011), que nunca un escrito sale "naturalmente bien" en un primer intento.

En este sentido, lxs estudiantes debieron producir textos propios a partir de consignas específicas. Si bien las actividades relativas a la escritura no fueron evaluadas con un criterio de evaluación clásico medible en términos de aprobación o desaprobación, cada uno de los textos elaborados fue corregido por el equipo docente. La devolución de los textos implicó en algunas ocasiones su reescritura, de acuerdo con el tenor y la cantidad de cuestiones a mejorar.

Algunos ejercicios de escritura fueron presenciales y otros domiciliarios. Las consignas que se propusieron fueron las siguientes: en primer lugar, responder preguntas ligadas a los textos trabajados en clase y entrenar la práctica del resumen; en segundo lugar, realizar trabajos de producción de textos propios (la realización de un ejercicio de "imaginación sociológica"); en tercer término, ejercitar el desempeño en situaciones evaluativas presenciales con tiempo acotado a partir de un "simulacro de parcial”. En algunas comisiones se sugirieron otros ejercicios de escritura, que se sumaron a los ya mencionados.

También se realizaron ejercicios de corrección colectiva en el aula, siempre atendiendo a no estigmatizar a lxs autorxs, copiando párrafos del texto en el pizarrón (o retomándolos a través de presentaciones de PowerPoint), y, en algunos casos, retomando ejercicios de escritura propuestos en el cuadernillo de trabajo. Las producciones se corrigieron teniendo en cuenta las siguientes cuestiones: la claridad de las ideas expresadas, el reflejo de una cabal comprensión de la consigna, la sintaxis, la concordancia en los tiempos verbales, la utilización de los signos de puntuación, la extensión de las oraciones y de los párrafos, y problemas frecuentes, tales como el uso de coloquialismos, las redundancias, la hipersubordinación y el exceso de tecnicismos.

En todas las ediciones del curso lxs docentes observamos que la escritura académica fue mejorando en el transcurso de las sucesivas entregas y a partir de las devoluciones realizadas. Asimismo, en algunos casos se llevaron a cabo talleres de escritura en el aula (explicitando qué se esperaba de un resumen, las estrategias de redacción, el ordenamiento de las ideas, el empleo de los signos puntuación, etc.). Sin embargo, desde el equipo también se observó la necesidad de profundizar y dar continuidad al trabajo sobre algunos casos que presentan importantes dificultades de escritura, que resultan imposibles de saldar en el mes de trabajo que propone el curso. 
Asimismo, lxs docentes solicitamos a quienes ingresaban que realizaran la entrega de al menos uno de los ejercicios escritos vía correo electrónico. De ese modo procuramos el entrenamiento en la práctica de enviar un correo "formal" a un/a docente, así como también el uso asiduo del correo en sí mismo (un medio de comunicación que será central a lo largo de sus futuras carreras como estudiantes).

Cabe señalar que las técnicas de división de grupos fueron importantes para favorecer la rotación, la circulación de la palabra y para evitar que en el aula cristalizaran tempranamente grupos homogéneos hacia su interior.

Otra innovación de las últimas ediciones del Curso consistió en la planificación de "semanas temáticas". En este sentido, la planificación establecida por el cronograma del curso dispuso la confluencia de distintas actividades vinculadas a temáticas, tales como géneros y memoria, en semanas específicas. Con ello buscamos articular el trabajo áulico con las propuestas elaboradas por distintos actores y espacios de la facultad, como parte de una política institucional común.

A modo de cierre de la cursada, lxs estudiantes de cada comisión trabajaron durante dos encuentros de la última semana en la elaboración de un afiche que condensó los principales ejes abordados, a partir de la revisión de actividades y contenidos discutidos durante el mes. En el último día del curso, las comisiones presentaron los trabajos a sus compañerxs de cada banda horaria reunidos en un aula única, lo cual propició el cierre a partir de un intercambio general sobre las producciones. La actividad fue presenciada, a su vez, por parte del equipo docente de Sociología General -cátedra central en el primer año de las carreras de Sociología- y por la coordinadora del curso. En este sentido, entendemos que, además de una primera instancia de articulación "hacia atrás" entre las trayectorias educativas previas y el ingreso a la educación universitaria, es necesario trabajar sobre una segunda instancia de coordinación "hacia adelante": la que existe entre el Curso Introductorio y las cátedras del primer año de la carrera.

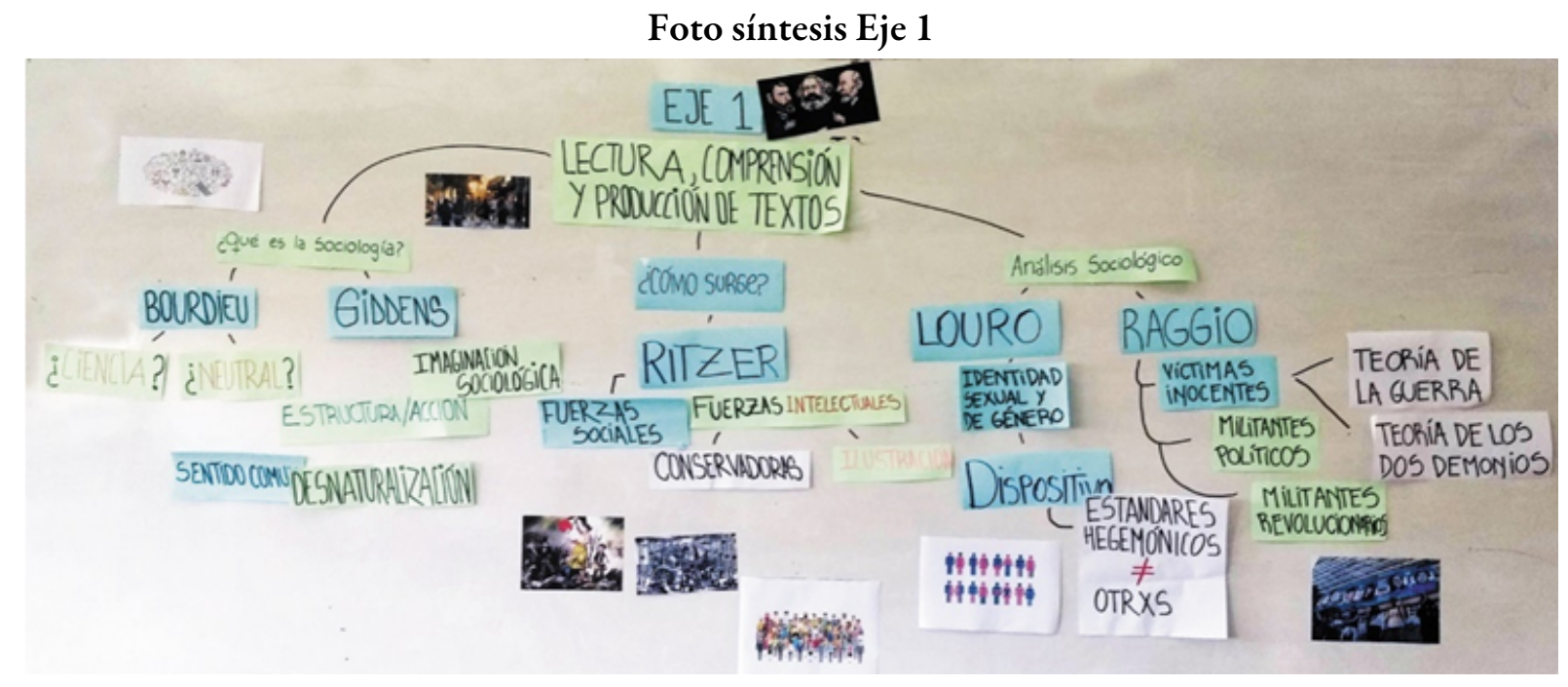

\subsection{El abordaje de la ambientación a la vida universitaria, del gobierno universitario y de la participación político-institucional estudiantil}

Hemos señalado que el ingreso al ámbito universitario también se encuentra estrechamente ligado a explicitar una serie de normas, prácticas, saberes vinculados al saber hacer y sentidos propios de la academia. Por este motivo, los ejes "ambientación a la vida universitaria" y "gobierno universitario y participación político-institucional estudiantil” se tornan sumamente importantes. 
En este sentido, a partir de estos ejes buscamos trabajar en dos direcciones. En primer lugar, planteamos la necesidad de brindar una batería de herramientas (administrativas, institucionales, gremiales y espaciales) a los lxs ingresantes, que resultaran necesarias para dar sus primeros pasos por la FaHCE. En esta línea, los talleres a cargo del Centro de Estudiantes, la Secretaría de Asuntos Estudiantiles y la Comisión de Estudiantes de Sociología brindaron información para la inscripción a las materias del primer año, sobre formas de acceder a los servicios de Bienestar Universitario (becas, comedor, boleto, albergue, servicios, entre otras), y sobre el uso del espacio de la biblioteca y la sala de informática. Asimismo, se buscó socializar el conocimiento sobre ordenanzas y reglamentos internos de la institución, tales como el Régimen de Enseñanza y Promoción, el Protocolo ante situaciones de violencia de géneros y la Ordenanza sobre lenguaje no binario.

En segundo lugar, en el marco del curso tuvo lugar una serie de actividades y talleres propias del ethos institucional de la Facultad de Humanidades y Ciencias de la Educación, y de la Universidad a la que pertenece. Por un lado, se llevó adelante un taller de géneros para introducir a lxs estudiantes en una cultura institucional con perspectiva de géneros. Por otro lado, se realizó un recorrido guiado por el predio en donde se encuentra emplazada la FaHCE-el exBatallón de Infantería de Marina 3-, que funcionó como centro clandestino de detención durante la última dictadura cívico-eclesiástico-militar en el país-, con el fin de conocer la historia y memoria desde un enfoque de los Derechos Humanos. Por último, en este mismo sentido, se desarrolló una clase teórica dictada por Aníbal Viguera, profesor titular de la cátedra Análisis de la Sociedad Argentina (asignatura perteneciente al plan de estudios de las carreras de Sociología), sobre la dictadura de 1976-1983. Esta actividad presenta un objetivo triple: por un lado, se conecta con la propuesta de visita guiada por el exBIM3, y, en ese sentido, se enmarca en las políticas de memoria y derechos humanos; por otro, busca introducir a lxs estudiantes en la dinámica propia de una clase teórica-expositiva; finalmente, se propone ejercitar la toma de apuntes. En función de ello, en los encuentros anteriores a la clase se anticiparon algunas herramientas para la toma de apuntes, mientras que en los encuentros siguientes se trabajó sobre los apuntes realizados, a partir de mapas conceptuales que reunían las principales ideas y de correcciones cruzadas entre lxs ingresantes.

Cabe destacar que todas instancias se realizaron en estrecha coordinación con los diversos actores institucionales que conforman la FaHCE, lo que permitió socializar la diversidad de voces y espacios que se desempeñan en la Facultad.

\section{Foto síntesis Eje 2}

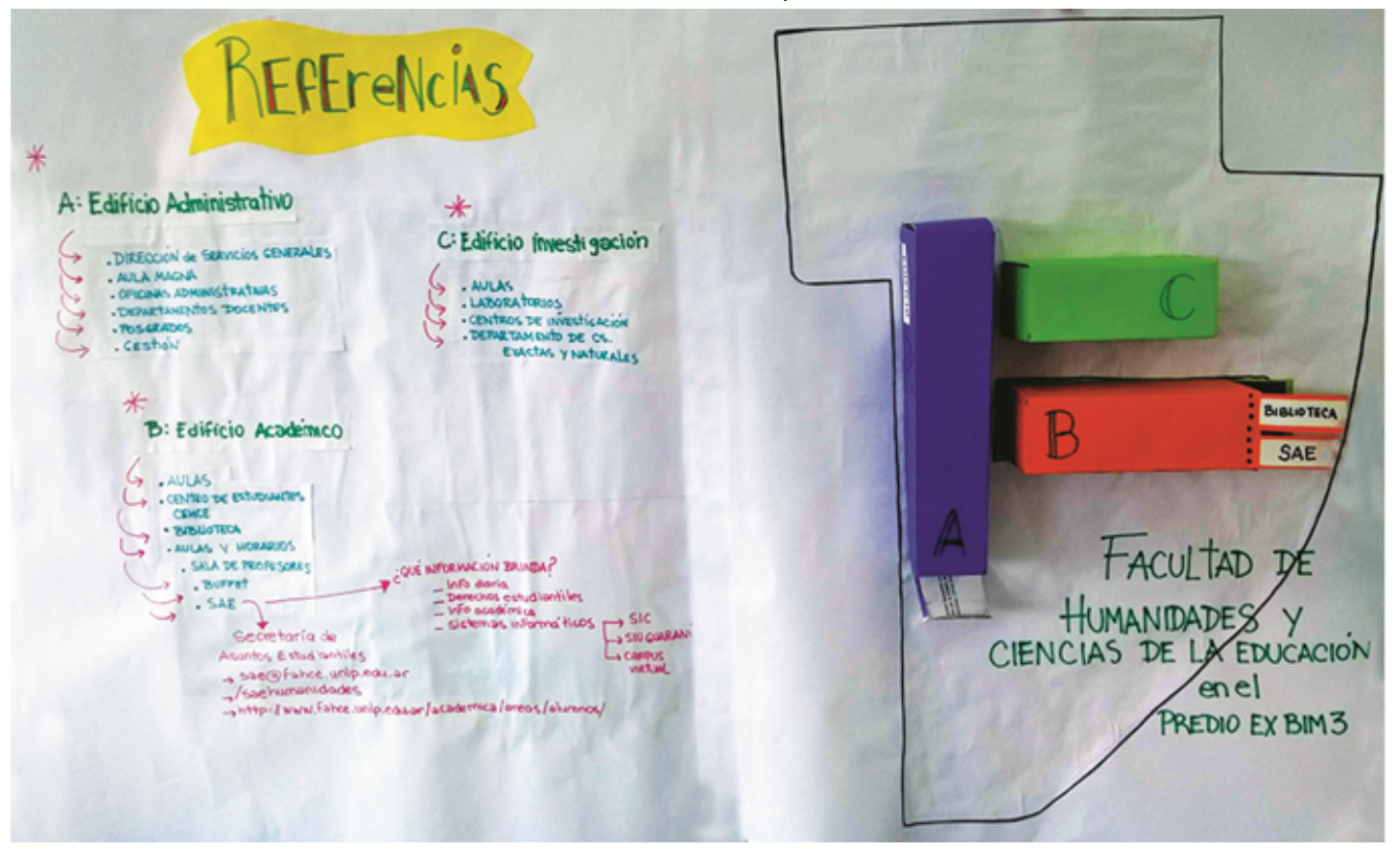




\subsection{Abordaje del Plan de estudios de la carrera e inserción ocupacional de lxs sociólogxs}

La primera parte de este eje, referida al abordaje del Plan de estudios, fue desarrollada en la actividad organizada por la Comisión de Estudiantes de Sociología. Por otro lado, para presentar el tema de la inserción ocupacional de lxs sociólogxs se organizaron tres paneles con 15 graduadxs de nuestras carreras, quienes comentaron sus recorridos profesionales. Uno de los objetivos de la charla fue que lxs estudiantes pudieran realizar preguntas acerca de su inserción ocupacional. De ese modo, se reunieron las comisiones según las tres franjas horarias, por lo cual quedaron conformadas tres charlas (dos por la mañana y una por la tarde). Las trayectorias laborales de lxs colegas que participaron del panel son variadas: se presentaron sociólogxs que trabajan en el Estado (municipal, provincial o nacional), en la Universidad (con trayectorias en docencia, investigación y extensión), en docencia terciaria y secundaria, en organizaciones no gubernamentales, y en consultoras. De manera previa al encuentro se brindó a lxs colegas una guía de preguntas para orientar sus intervenciones en el panel, que fue a su vez moderado por lxs docentes del curso. Asimismo, se procuró dar lugar al intercambio, a las preguntas y comentarios por parte de lxs ingresantes, quienes en cada edición valoraron de manera positiva esta instancia, en tanto la destacaban como un ejercicio de anticipación capaz de reducir la ansiedad sobre posibles futuros laborales que muchas veces les resultaban demasiado inciertos por no tratarse de una de las carreras universitarias tradicionales.

\section{CONCLUSIONES. Preguntas y desafíos Pendientes}

Me gustó el lugar desde donde se da el curso. Fue la primera vez (yo estudio en otra facultad) que me sentí parte de la UNLP (ingresante a Sociología, marzo de 2019)

A modo de cierre, nos gustaría recuperar algunas de las cuestiones presentadas, así como también dejar planteadas algunas preguntas y desafíos pendientes. Por una parte, consideramos que el curso es un aporte fundamental en relación con la igualación de las trayectorias en el primer año. Esta afirmación se desprende de tres apreciaciones: 1 ) de las valoraciones de lxs estudiantes; 2) de las observaciones de los equipos docentes, que evidencian una notable mejora en la escritura de lxs ingresantes, y 3) de la conformación de grupos de pares entre lxs estudiantes, lo cual fortalece redes de sociabilidad que son fundamentales en una instancia de transición como es el ingreso a la universidad, y repercuten de manera positiva en la filiación institucional.

Con respecto a los desafíos pendientes, en cada edición del curso detectamos casos sumamente críticos en lo referido a la escritura. Se trata de situaciones de estudiantes que presentan una distancia notoria con respecto al tipo de escritura académica que se espera y se promueve desde la universidad. A partir de este balance, si bien el trabajo en el curso contribuye a que la escritura mejore en todos los casos, muchas veces no es suficiente. Si se considera lo antedicho, un trabajo de seguimiento durante todo el primer año podría contribuir al propósito de acercarse a un piso común.

Por otro lado, como indican investigaciones recientes sobre el ingreso a los estudios superiores, los dispositivos pensados para actuar sobre un momento acotado en la instancia de ingreso a una nueva institución educativa presentan limitaciones en relación con su impacto. En ese sentido, consideramos que es importante la profundización de la vinculación del espacio del curso con las materias que se cursan en el primer año, es decir, casi inmediatamente (semanas después) a la finalización del curso. Si bien realizamos actividades de articulación (como la mencionada en relación con materias troncales del primer año), resulta imprescindible profundizar los puentes, no ya "hacia atrás" -entre la escuela secundaria y el ingreso a la universidad-, sino también "hacia delante" -entre los cursos de ingreso y las materias de primer año-. De este modo se propiciaría una sinergia en la cual, por un lado, el curso retomaría actividades propias de las cátedras (lo que evitaría que se transformase en una "isla" que infantilice a quienes acceden al nivel superior), 
y, por otro, las cátedras retomarían actividades propias del curso (referidas a la visibilización de prácticas y supuestos que no todxs conocen por igual).

En suma, consideramos que el curso es un aporte fundamental tanto en lo referido a la construcción de una primera aproximación hacia una mirada sociológica como hacia la socialización en la vida universitaria, las redes de sociabilidad y la construcción del oficio de estudiante universitarix. En ese sentido, se trata de un espacio favorable al desarrollo de una "alfabetización académica" tendiente a garantizar el derecho a la educación superior.

\section{ReFERENCIAS}

Aréchaga, A. J., Beliera, A., Boix, O., Corsiglia Mura, L., Cueto Rúa, S., Di Piero, E., Galar S., y Gubilei, E. (2014). Puentes hacia la inclusión universitaria. Un análisis a partir de la experiencia en el Curso de Ingreso a las carreras de Sociología de la FaHCE-UNLP (2012 - 2014). Ponencia presentada en VIII Jornadas de Sociología de la Universidad Nacional de La Plata, La Plata, Argentina.

Becker, H. (2011) Manual de escritura para cientificos sociales. Cómo empezary terminar una tesis, un libro o un artículo. Buenos Aires: Siglo XXI Editores.

Borges, J.L. (1985). Discusión. En Obras Completas, Volumen I, 90- 112. Editorial Emecé.

Carlino, P. (2005). Escribir, leer, y aprender en la universidad. Una introducción a la alfabetización académica. Buenos Aires: Fondo de Cultura Económica.

Chiroleu, A. (1998). Acceso a la universidad: sobre brújulas y turbulencias. Pensamiento Universitario, 6 (7), 1-11.

Cotignola, M., Legarralde, M., y Margueliche, J. C. (2017). Las trayectorias universitarias de estudiante de Sociología de la FaHCE. Un análisis desde los registros administrativos. Cuestiones de Sociología, (17), e045.

Coulon, A. (1995). Etnometodología y Educación. Argentina: Paidós

Di Piero, E. (2015). ¿Mérito y azar?: nociones de justicia distributiva y selección soft. El caso de una escuela secundaria tradicional frente al mandato incluso (Tesis de maestría). FLACSO. Sede Académica Argentina, Buenos Aires, Argentina.

Di Piero, E. (2019). Articulaciones nivel secundario-nivel superior: una tipología de las politicas de admisión a las carreras de Sociologia en el país y de las expectativas de jóvenes ingresantes a las carreras de Sociología en la Facultad de Humanidades y Ciencias de la Educación - Universidad Nacional de La Plata en el año 2018. Ponencia presentada en VI Reunión Nacional de Investigadorxs en Juventudes de Argentina, Córdoba, Argentina.

Ezcurra, A. M. (2011). Igualdad en educación superior. Un desafío mundial. Buenos Aires: Universidad Nacional de General Sarmiento.

Pierella, M. P. (2018). El primer año de universidad desde la perspectiva de los profesores. Políticas de recepción, enseñanza y currículum. Espacios en Blanco. Revista de Educación, 28, 161-181.

Rossi Case, L., Varela, S., y Aguiar, D. (2006). Estudio sobre los ingresantes a la carrera de Sociología de la UNLP en el año 2004. Caracterización del perfil socio-cultural y su evolución en los últimos años. Cuestiones de Sociología, 2006, (3), 316-340.

Suasnábar, C., y Rovelli, L. (2015). Ampliaciones y desigualdades en el acceso y egreso de estudiantes a la Educación Superior en la Argentina. Pro-Posiçoes, 27 (3), 81-104.

Terigi, F. (2009). Las trayectorias escolares, del problema individual al desafio de politica educativa. Buenos Aires: Ministerio de Educación de la Nación.

\section{Notas}

1 En el caso argentino, a diferencia de lo que ocurre en la mayor parte de los países del mundo, no existen mayoritariamente exámenes de ingreso. De ese modo, se produce una selección educativa sutil, implícita o soft, que, aunque implique una democratización imperfecta, es siempre preferible a la eliminación directa vía examen (Di Piero, 2015). 
2 Juan, ingresante a Sociología 2020, 20 años.

3 La edición 2020 del cuadernillo del Curso Introductorio a las carreras de Sociología se encuentra disponible en: http:/ /www.fahce.unlp.edu.ar/academica/areas/sociologia/descargables/cuadernillo-2020 